\title{
Lösungen anbieten
}

\author{
Interview mit Oliver Theißen, Hamburg - Das Gesund- \\ heitswesen ist im Umbruch. Pflegekräfte und Pflegeeinrich- \\ tungen müssen sich ständig auf Neuerungen einstellen. Unter- \\ stützung erhalten sie dabei von der Standard Systeme GmbH, die \\ Prozesse begleitet und Lösungen für den Pflegealltag entwickelt.
}

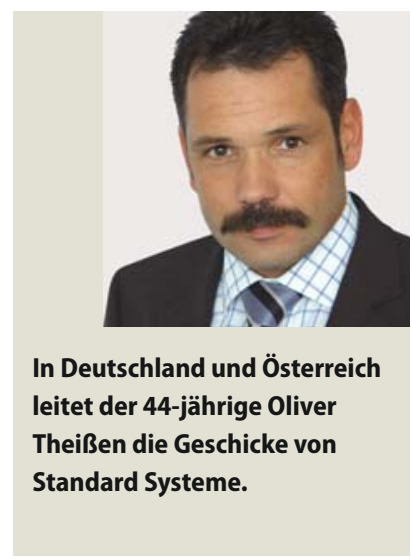

Heilberufe: Herr Theißen, pflegen heißt heute auch immer dokumentieren. Viele Pflegekräfte sehen diese Aufgabe als lästige Pflicht. Was antworten Sie darauf?

Theißen: Dokumentation gehört in jedem Beruf dazu. Eine Pflegedokumentation macht dann Sinn, wenn sie die tägliche pflegerische Arbeit erkennbar werden lässt und somit auch deutlich wird, dass die Bedürfnisse der Pflegebedürftigen berücksichtigt wurden. Letztendlich wird bei einer gut geführten Dokumentation die Ergebnisqualität des jeweiligen Pflegebedürftigen visualisiert.

Was leistet Pflegedokumentation noch, wohin geht die Entwicklung?

\section{- Das Unternehmen}

Seit 35 Jahren ist die Hamburger Standard Systeme GmbH auf dem deutschen Markt vertreten. Das dänische Mutterunternehmen wurde bereits 1962 gegründet. Mittlerweile existieren sechs nationale Gesellschaften mit 30 Firmen und circa 250 Mitarbeitern. Von der manuellen oder elektronischen Pflegedokumentation, über Organisationslösungen, Unternehmensberatung bis zur Objekteinrichtung finden Unternehmen des Gesundheits- und Sozialwesens alles, was sie im Organisationsbereich benötigen. Man versteht sich als Mehrwertunternehmen, in dem Beratung und Service selbstverständlich dazugehören. Nicht ohne Grund stammen alle Fachberater aus der Pflegebranche.
In der Dokumentation wird die Philosophie, die Konzeption, das Menschenbild und das Unternehmensmanagement erkennbar. Ebenso zeigt sich, ob bereits neue pflegewissenschaftliche Erkenntnisse umgesetzt sind wie beispielsweise die Expertenstandards. In den Einrichtungen werden heute verstärkt Lösungen entwickelt, die die Pflegedokumentation optimieren sollen.

\section{Seit 35 Jahren unterstützt Standard} Systeme mit seinem Angebot Pflegeeinrichtungen. Welche Veränderungen hat Ihr Unternehmen in dieser Zeit begleitet? Die Veränderungen waren gerade in den letzten Jahren sehr vielfältig. Die wichtigsten Veränderungen ergeben sich aufgrund von Gesetzesänderungen oder -überarbeitungen wie der Einführung und Veränderung des Pflegeversicherungsgesetzes, der Novellierung des Heimgesetzes, der Überleitung des BSHG in das SGB XII, der Einführung von SGB IX und SGB V und Veränderungen im Betreuungsgesetz - nicht zuletzt aber auch durch die Einführung der DRGs und aktuelle Urteile mit Einfluss auf die Pflegedokumentation.

Veränderte Bedürfnisse der Einrichtungen erfordern Lösungen. Wie reagiert lhr Unternehmen darauf?

Vor 35 Jahren wurde vielfach noch mit einem Lochkarten- oder Kadexsystem gearbeitet. Heute werden von uns modernste Produkte zur Optimierung der Dokumentation eingesetzt - Map- pensysteme, Visitenwagen, Signalreiter. Darüber hinaus wird die elektronische Dokumentation immer wichtiger. Mittlerweile bieten wir ein elektronisches Dokumentationssystem mit allen denkbaren Schnittstellen an, zum Beispiel für die elektronische Wunddokumentation, die Dienstplanung oder die Heimverwaltung. Modernste Druckmaschinen sorgen dafür, dass wir die vielen Druckaufträge auch zeitnah erledigen können. Vor 35 Jahren gab es noch keine Seminarcenter - heute nicht mehr vorstellbar. Hier bieten wir eine Vielzahl von aktuellen Veranstaltungen an. Auch durch die Gründung einer Unternehmensberatung sind wir den wachsenden Anforderungen an die Qualitätssicherung gerecht geworden.

\section{Standard Systeme hat in verschiedenen} Städten Arbeitskreise gegründet, in denen sich Pflegeprofis austauschen können. Welche Idee steckt dahinter? Die Arbeitskreise wenden sich an Pflegedienstleitungen und Qualitätsbeauftragte. Sie bieten die Möglichkeit, sich über aktuelle Fragen regional auszutauschen. Gleichzeitig erfahren die Teilnehmer, wie die jeweiligen Themenkomplexe in den einzelnen Einrichtungen umgesetzt werden sollen. Darüber hinaus hat sich am 4. Dezember 2007 das bundesweite intersektorale Netzwerk gegründet, das sich über aktuelle gesundheitspolitische Fragen austauscht.

Vielen Dank für das Gespräch. 